\title{
An optimization method for dynamics of structures with repetitive component patterns
}

\author{
D. Akçay Perdahcioğlu • M. H. M. Ellenbroek • \\ P. J. M. van der Hoogt • A. de Boer
}

Received: 18 August 2008 / Revised: 24 February 2009 / Accepted: 25 April 2009 / Published online: 19 May 2009

(C) The Author(s) 2009. This article is published with open access at Springerlink.com

\begin{abstract}
The occurrence of dynamic problems during the operation of machinery may have devastating effects on a product. Therefore, design optimization of these products becomes essential in order to meet safety criteria. In this research, a hybrid design optimization method is proposed where attention is focused on structures having repeating patterns in their geometries. In the proposed method, the analysis is decomposed but the optimization problem itself is treated as a whole. The model of an entire structure is obtained without modeling all the repetitive components using the merits of the Component Mode Synthesis method. Backpropagation Neural Networks are used for surrogate modeling. The optimization is performed using two techniques: Genetic Algorithms (GAs) and Sequential Quadratic Programming (SQP). GAs are utilized to increase the chance of finding the location of the global optimum and since this optimum may not be exact, SQP is employed afterwards to improve the solution. A theoretical test problem is used to demonstrate the method.
\end{abstract}

D. Akçay Perdahcıoğlu (凶) · M. H. M. Ellenbroek ·

P. J. M. van der Hoogt - A. de Boer

Department of Applied Mechanics, University of Twente,

7500AE Enschede, The Netherlands

e-mail: d.akcay@ctw.utwente.nl

M. H. M. Ellenbroek

e-mail: m.h.m.ellenbroek@ctw.utwente.nl

P. J. M. van der Hoogt

e-mail: p.j.m.vanderhoogt@ctw.utwente.nl

A. de Boer

e-mail: a.deboer@ctw.utwente.nl
Keywords Hybrid design optimization method • Repetitive patterns - Component mode synthesis • Backpropagation neural networks • Genetic algorithms $\cdot$ Sequential quadratic programming

\section{Introduction}

Currently the design of structures such as cars, aircrafts and aerospace applications are analyzed extensively using Finite Element (FE) methods, possibly years before the first prototype is built. The benefits of the FE method may include increased accuracy, a faster and less expensive design cycle and a better comprehension of the structural behavior which makes it an indispensable tool for a complicated engineering analysis. Investigation of the structural behavior under static loads often requires very fine meshes. On the other hand, investigation of the dynamic properties of these structures requires only a few deformation modes. These could be calculated with coarse meshed FE models which are less time consuming. Instead of creating a new coarse mesh for structural dynamic analysis, it is possible to keep the fine mesh of the static analysis but decrease the computation time by employing a suitable reduction method. The main assumptions when building reduction methods are the existence of an accurate FE model and the restrictions on the frequency range. In large projects (e.g. an aircraft design), reducing the complete model is still a cumbersome task. That is why the tendency is to divide the analysis of such models into several parts, construct separate models for each part and then use these models to reconstruct the whole model. This process is called substructuring. The so-called Component Mode Synthesis (CMS) 
technique is a substructuring method which has been utilized since 1960s for the dynamic analysis of complex structures. The idea behind this technique is to divide the structure into a number of substructures, calculate the corresponding reduced order FE models and then assemble them to obtain a reduced order FE model of the complete structure. This technique is commonly preferred in industry because it allows modeling of each substructure by different design groups. Any design change in a single substructure affects only the system matrices of that substructure. Hence, if a modification is required in any specific substructure (e.g. the solid rocket boosters of a space shuttle or the interstage of a launcher), only the system matrices of that particular substructure are changed and coupled with the rest of the already analyzed substructures. This saves much valuable computation time. Some structures involve repeating patterns in their geometry e.g. the wings of a plane, one cyclic sector of an industrial blisk, a bladed disk, etc. Modeling one repeating component and utilizing its system matrices for the remaining identical parts is another advantage of CMS.

One of the more common structural problems encountered in dynamic analyses is resonance. Resonance may cause large strains and large stresses in a structure which can lead to failure by fatigue. In most situations it is not possible to control the frequency content of the external loads. Therefore resonance can only be avoided by changing the design so as to keep the resonance frequencies away from the excitation frequency. In reality there are always other factors that have to be considered besides shifts in the resonance frequencies. These might be additional constraints coming from practical design and performance requirements such as minimum total mass, effect of the modifications on the other dynamic properties, restrictions on the geometric properties of the structure such as bounded lengths or widths. Under the concept of design optimization, all these criteria can be tackled at the same time.

The objective of the current paper is to define a design optimization method for dynamic analyses of structures with repetitive component patterns, in which the benefits of CMS can be utilized to increase the computational efficiency of the optimization process.

For the optimization of complex structures many design parameters come into play due to multidisciplinary interactions. Then, optimization at a single level can be a computational burden. Decomposing a large optimization problem into smaller subproblems becomes necessary for solving such problems. These subproblems may represent either physical subsystems (components of a structure) or the disciplines. For decomposition, the design variables and the constraints need to be separated into smaller sized problems that are only weakly connected. In the MultiDisciplinary Optimization (MDO) field a two-stage solution is carried out: first, decomposed small-size problems are solved individually at the subsystem level. Then, these are coordinated so as to optimize the entire problem. The coordination level is usually referred to the top level (Sobieszczanski-Sobieski 1989). Therefore, optimization problems with a large number of design parameters could be effectively solved by defining small-size optimization problems.

For structural dynamics problems, application of MDO schemes is difficult. This is because the interface forces between components are frequency dependent and they may also strongly depend on the values of the component design variables. Additionally, the resonance frequencies are determined by the behavior of the entire structure.

In Hou et al. (1995), a design optimization method is defined where CMS is integrated into a two-level design optimization scheme. In the method, CMS is employed for the decomposition of the analysis as well as to define the subproblems. The static modes, the eigenvalues and the corresponding interface d.o.f of eigenvectors of the components are considered as the top level (intermediate) design variables. At the component (subsystem) level, the FE model properties of the components are treated as design variables. Each component is optimized locally, to satisfy the corresponding intermediate design variables defined by the top level, in order to improve the performance of the assembled structure. Issues concerning the accuracy of the results are pointed out in Hou et al. (1995). Another point that has to be taken into account is the detrimental effect of the intermediate design variables on the efficiency of the method, especially for the optimization of complex structures. The number of d.o.f at the interfaces of the components increases with the complexity of the problem, which may cause a drastic increase of the number of intermediate design variables. Thus, there is always a high chance that the number of intermediate design variables may exceed the total number of component design variables, which is contradictory with the purpose of a multilevel optimization scheme.

In Wind et al. (2008), a two-level scheme is presented. As in Hou et al. (1995), both the analysis and the optimization problem are decomposed using CMS. The main difference between the methods is that in Wind et al. (2008) the optimization is performed only at the component level and no optimization is performed at the top level. Each subproblem is the same of the main optimization problem, except that the design variables that do not correspond to the component of 
interest are fixed at constant values. Therefore, the intermediate design variables are eliminated to prevent the complications mentioned in Hou et al. (1995). In method of Wind et al., the top level is only used for the distribution of the component level solutions to each subproblem. The drawback of this strategy is the possibility of convergence problems especially when the design variables of one component depend on the design variables of the other components (Wind 2005).

In the current paper, CMS is only used for the decomposition of the analysis, but the optimization problem itself is treated as a whole with the purpose of avoiding the above mentioned complications due to the decomposition. The numerical techniques that are used in their primitive forms in Wind et al. (2008) such as Backpropagation Neural Networks (BNN) and Genetic Algorithms (GAs) are extended with more robust techniques for preventing over-fitting problems (see Section 3), and effective handling of linear and nonlinear constraints (see Section 4). Moreover, additional numerical techniques are employed for the sake of improving the solution quality and robustness. A theoretical test problem was selected in the direction of emphasizing the disregarded attributes of CMS in Wind et al. (2008) i.e. repeating component patterns for effective structural optimization.

This paper is built up as follows: in Section 2, Component Mode Synthesis (CMS) and the Craig-Bampton method are explained in detail. In Sections 3 and 4, Neural Network (NN) surrogate models and the employed optimization strategies, Genetic Algorithms (GAs) and Sequential Quadratic Programming (SQP) are described. The proposed optimization method is introduced in Section 5. Next, the method is demonstrated for a theoretical test problem and finally in Section 7, conclusions are presented.

\section{Component mode synthesis and Craig-Bampton method}

CMS involves breaking up a large structure into several substructures (components), obtaining reduced order system matrices of each component and then assembling these matrices to attain reduced order system matrices of the entire structure. All substructure calculations are independent of each other, and therefore a design change in one component has no effect on the models of the other components.

Let us assume that a FE model of a structure is constructed on a domain $\Omega$ and is divided into $N$ nonoverlapping substructures such that each component is defined on the sub-domain $\Omega^{c}$. Thus, excepting the nodes on the interface boundaries, each node belongs to one and only one component. The linear dynamic behavior of an undamped component, labeled c, is governed by the equations,

$\mathbf{M}^{c} \ddot{\mathbf{u}}^{c}+\mathbf{K}^{c} \mathbf{u}^{c}=\mathbf{f}^{c}+\mathbf{g}^{c} \quad c=1,2, \ldots, N$

where $\mathbf{M}^{c}, \mathbf{K}^{c}$ and $\mathbf{u}^{c}$ are the mass matrix, stiffness matrix and vector of local d.o.f of the component, respectively. The vector $\mathbf{f}^{c}$ represents the external loads, and the vector $\mathbf{g}^{c}$ represents the interface forces between the component $c$ and the neighboring components, that ensure dynamic equilibrium at the interfaces. The partitioned form of (1) can be written as follows:

$$
\left[\begin{array}{ll}
\mathbf{M}_{i i}^{c} & \mathbf{M}_{i b}^{c} \\
\mathbf{M}_{b i}^{c} & \mathbf{M}_{b b}^{c}
\end{array}\right]\left\{\begin{array}{c}
\ddot{\mathbf{u}}_{i}^{c} \\
\ddot{\mathbf{u}}_{b}^{c}
\end{array}\right\}+\left[\begin{array}{cc}
\mathbf{K}_{i i}^{c} & \mathbf{K}_{i b}^{c} \\
\mathbf{K}_{b i}^{c} & \mathbf{K}_{b b}^{c}
\end{array}\right]\left\{\begin{array}{c}
\mathbf{u}_{i}^{c} \\
\mathbf{u}_{b}^{c}
\end{array}\right\}=\left\{\begin{array}{c}
\mathbf{f}_{i}^{c} \\
\mathbf{f}_{b}^{c}
\end{array}\right\}+\left\{\begin{array}{c}
\mathbf{g}_{i}^{c} \\
\mathbf{g}_{b}^{c}
\end{array}\right\}
$$

where $i$ and $b$ refer to interior and boundary, respectively.

It has already been discussed that in dynamic analyses it is not necessary to use the information of all the d.o.f. Thus, in CMS, in order to reduce the structure model, nodal displacement vectors $\mathbf{u}^{c}$ of each substructure are replaced by their approximations. This is done by searching for approximate solutions in a smaller space spanned by the columns of a transformation matrix $\mathbf{T}^{c}$, such that

$$
\left\{\begin{array}{c}
\mathbf{u}_{i}^{c} \\
\mathbf{u}_{b}^{c}
\end{array}\right\} \approx \mathbf{T}^{c}\left\{\mathbf{q}^{c}\right\}
$$

where $\mathbf{q}^{c}$ is a vector of generalized coordinates and $\operatorname{dim}\left(\mathbf{q}^{c}\right) \ll \operatorname{dim}\left(\mathbf{u}^{c}\right)$. $\mathbf{T}^{c}$ is defined by a reduction basis.

In the Craig-Bampton method (Craig and Bampton 1968), the reduction basis is obtained by utilizing the fixed interface normal modes and the constraint modes of each component.

The fixed interface normal modes are calculated by restraining all d.o.f. at the interface and solving the eigenvalue problem:

$$
\left(\mathbf{K}_{i i}^{c}-\omega_{j}^{2} \mathbf{M}_{i i}^{c}\right)\left\{\boldsymbol{\phi}_{i}^{c}\right\}_{j}=0 \quad j=1,2, \ldots, F
$$

where $\omega_{j},\left\{\boldsymbol{\phi}_{i}^{c}\right\}_{j}$ are the eigenvalue and the corresponding eigenvector of the $j^{\text {th }}$ normal mode respectively, and, $\mathrm{F}$ is the number of truncated normal modes. The fixed interface normal modes of a component $c$ are:

$$
\begin{aligned}
\boldsymbol{\phi}^{c} & =\left[\begin{array}{cccc}
\left\{\boldsymbol{\phi}_{i}^{c}\right\}_{1} & \left\{\boldsymbol{\phi}_{i}^{c}\right\}_{2} & \ldots & \left\{\boldsymbol{\phi}_{i}^{c}\right\}_{F} \\
\mathbf{0}_{b} & \mathbf{0}_{b} & \ldots & \mathbf{0}_{b}
\end{array}\right] \\
& =\left[\begin{array}{c}
\boldsymbol{\phi}_{j} \\
\mathbf{0}_{b}
\end{array}\right]^{c} \quad j=1,2, \ldots, F .
\end{aligned}
$$


The constraint modes are calculated by statically imposing a unit displacement to the interface d.o.f. one by one while keeping the displacement of other interface d.o.f. zero and the interior d.o.f. of the substructure force free, such that

$$
\left[\begin{array}{cc}
\mathbf{K}_{i i}^{c} & \mathbf{K}_{i b}^{c} \\
\mathbf{K}_{b i}^{c} & \mathbf{K}_{b b}^{c}
\end{array}\right]\left[\begin{array}{c}
\boldsymbol{\psi}_{i b}^{c} \\
\mathbf{I}_{b b}^{c}
\end{array}\right]=\left\{\begin{array}{c}
\mathbf{0}_{i b}^{c} \\
\mathbf{R}_{b b}^{c}
\end{array}\right\}
$$

where $\mathbf{R}_{b b}^{c}$ is the unknown reaction force vector. The constraint mode matrix $\psi$ of component $\mathrm{c}$ is defined as:

$\boldsymbol{\psi}^{c}=\left[\begin{array}{c}\boldsymbol{\psi}_{i b}^{c} \\ \mathbf{I}_{b b}\end{array}\right]=\left[\begin{array}{c}-\mathbf{K}_{i i}^{c-1} \mathbf{K}_{i b}^{c} \\ \mathbf{I}_{b b}\end{array}\right]$.

Therefore, the Craig-Bampton transformation matrix $\mathbf{T}_{C B}^{c}$ for component $\mathrm{c}$ is

$$
\mathbf{T}_{C B}^{c}=\left[\begin{array}{ll}
\boldsymbol{\phi}_{j} & \boldsymbol{\psi}_{i b} \\
\mathbf{0}_{b} & \mathbf{I}_{b b}
\end{array}\right]^{c}
$$

and the Craig-Bampton reduced stiffness and mass matrices are given by: $\mathbf{K}_{C B}^{c}=\mathbf{T}_{C B}^{c}{ }^{T} \mathbf{K}^{c} \mathbf{T}_{C B}^{c}$, $\mathbf{M}_{C B}^{c}=\mathbf{T}_{C B}^{c}{ }^{T} \mathbf{M}^{c} \mathbf{T}_{C B}^{c}$, respectively. The external loads and the internal forces are $\mathbf{f}_{C B}^{c}=\mathbf{T}_{C B}^{c}{ }^{T} \mathbf{f}^{c}, \mathbf{g}_{C B}^{c}=\mathbf{T}_{C B}^{c}{ }^{T} \mathbf{g}^{c}$, respectively.

After reducing the system matrices of each substructure, the next step is the assembly of all these matrices. The substructures can be interpreted as macro elements for the assembly. The local reduced d.o.f $\mathbf{q}^{c}$ of a component $c$ is related to the reduced d.o.f $\mathbf{u}_{s}$ of the entire structure by

$\mathbf{q}^{c}=\left\{\begin{array}{c}\eta^{c} \\ \mathbf{u}_{b}^{c}\end{array}\right\}=\mathbf{B}^{c} \mathbf{u}_{s}$.

The matrix $\mathbf{B}^{\mathbf{c}}$ is a Boolean matrix which relates the boundary d.o.f $\mathbf{u}_{b}^{c}$ and the interior generalized d.o.f $\eta^{c}$ of component $c$ to the corresponding d.o.f of the entire structure. Hence, (9) is a compatibility condition between each substructure $c, c=1,2, \ldots, N$ and the structure. Using this condition, component equations (1) including the Craig-Bampton reduced system matrices can be assembled as

$\mathbf{M}_{s} \ddot{\mathbf{u}}_{s}+\mathbf{K}_{s} \mathbf{u}_{s}=\mathbf{f}_{s}$

where

$$
\begin{aligned}
\mathbf{M}_{s} & =\sum_{c=1}^{N} \mathbf{B}^{c^{T}} \mathbf{M}_{C B}^{c} \mathbf{B}^{c}, \mathbf{K}_{s}=\sum_{c=1}^{N} \mathbf{B}^{c^{T}} \mathbf{K}_{C B}^{c} \mathbf{B}^{c}, \\
\mathbf{f}_{s} & =\sum_{c=1}^{N} \mathbf{B}^{c^{T}} \mathbf{f}_{C B}^{c}
\end{aligned}
$$

are the reduced mass and stiffness matrices and the external load vector of the entire structure respectively.
It is important to point out that the interface forces $\mathbf{g}_{C B}^{c}$ are all cancelled out after assembly. This assembly where the substructures are assembled using the compatibility of the interface nodes is called primal assembly.

The compatibility of interface nodes for the assembly may cause trouble when the assembled substructures have non-matching interface nodes. Using the compatibility of interface forces between components is one way to tackle such a problem, as presented by Rixen (Rixen 2004).

\section{Neural network surrogate models}

It is becoming common practice to use surrogate models instead of FE models in most structural optimization problems. The main advantage of surrogate models is to reduce computation time as well as to make design optimization of complex structures possible. For surrogate modeling, firstly input-target pairs (training set) are required which are obtained by running a FE model for varying values of the design variable set. Then the relationship between these pairs is defined by a response surface which is called a surrogate model. Once the surrogate model is found, it replaces the FE model in the optimization problem.

In the current research, Backpropagation Neural Networks (BNNs) (Hagan et al. 1996) are employed for surrogate modeling with a structure defined as in Hornik (1989). The ability to approximate continuous and differentiable functions to any desired degree of accuracy makes them attractive tools for our purpose.

The working principle of NNs is the same as the Least Squares Method (LSM). First, the NNs are provided with a set of input-target pairs. The input pairs are applied to the network and the corresponding network outputs are obtained. Then these outputs are compared to the target values and the network parameters are adjusted in order to minimize the mean square error between the network output and the target values.

In the network structure, a set of nonlinear (sigmoid) transfer functions are employed for the learning process. Its number has a direct effect on the complexity of the surrogate model. The increasing number of these functions in the NN structure leads to highly nonlinear surrogate models which may cause over-fitting. Over-fitting occurs when the error on the training set is driven to a very small value but in the case of a new input-target pair involvement, the network becomes inadequate to predict the new situation. When there is no information about the complexity of the 
underlying behavior, the effective number of nonlinear transfer functions can't be estimated beforehand. Several techniques have been developed to determine that number without resorting to inefficient trial and error. In this study, the Bayesian regularization of Mackay (Mackay 1992) was used for the calculation of this number and preventing possible over-fitting problems. The algorithm defined in Foresee and Hagan (1997) was utilized for the training process.

\section{Optimization}

Many structural optimization problems require the solution of non-convex nonlinear optimization problems, where non-convexity may introduce multiple local optima. The pursuit of a global optimum is one of the main concerns of many researchers. Classical Nonlinear Programming (NLP) techniques may have the risk of being trapped in one of the local optima based on the selected initial point. Therefore, in the current method Sequential Quadratic Programming (SQP), a widely used classical NLP technique, is utilized in combination with a global optimization method, the Genetic Algorithms (GAs). The main reason to avoid using GA alone is that it lacks a convergence theory. Its solutions are based on estimations and might not be exact. Thus, GA is employed to provide an initial point for SQP and then SQP is called using that point to find an exact optimum solution. The justification of this choice is reported in Akçay Perdahcioğlu et al. (2007).

The GA algorithm employed handles nonlinear constraints differently than linear and bound constraints. Since a region restricted by bound and linear constraints defines a convex set, it is not difficult to satisfy these constraints by generating points in the feasible domain throughout the optimization process. Unfortunately this is not an easy task for nonlinear constraints, which is the reason why they are treated separately. The Composite Lagrangian Barrier-Augmented Lagrangian (CLB-AL) algorithm of Conn et al. (Conn et al. 1991; Gould et al. 1997) provides a framework for handling nonlinear constraints. The details of the GA algorithm employed can be found in Kumar (2007).

In SQP, an NLP problem is attempted to be solved using a sequence of Quadratic Programming (QP) subproblems. The construction of QP subproblems are the same for all SQP strategies. Available strategies only differ by selection of a QP solver and a merit function which promotes convergence from arbitrary starting points. In this study, the Null Space Active Set method of Gill et. al. (Gill et al. 1984) is used for solving QP subproblems. The merit function is selected as in Han (1977).

\section{The design optimization method}

The proposed design optimization method is illustrated in Fig. 1. In this method, the analysis is decomposed so that each component analysis is carried out separately. The optimization problem, however, is solved as a whole at the Structure Level.

The solution process starts with the problem analysis which firstly involves understanding the problem under consideration. Then, the components of the structure and the corresponding design variables are selected and the FE models of the components are parameterized for surrogate modeling based on the obtained observations. Finally, the objective and the constraint functions of the optimization problem are defined.

The second step in the method is the Design of Computer Experiments (DOCE). Here, a set of sample points is selected from the design space for surrogate modeling. Each sample point requires a new FE model run which means the number of the sample points may have drastic effects on the computation time. In most applications, the intention is to extract valuable information about the general trend with the smallest number of sample points. The required number of points depends strongly on the nonlinearity of the trend which is not easy to estimate beforehand. Therefore the method is initialized with a reasonable number of sample points and, if this set is not large enough to extract the general trend information then it is extended in the next iteration. In this research, Latin Hypercube Sampling (Giunta et al. 2003; McKay et al. 1979) is employed for sampling and the initial number of sample points are selected as 10 times the number of the total design variables.

Having generated the sample points, the required information for calculating the structure response is gathered at the Component Level. This level involves the parameterized FE models of the components where only one model is taken into account for the repeating geometries. The reduced system matrices of the components are calculated for each component configuration and stored in the component libraries. If there exist any components with no design variables, their reduced system matrices are calculated once and assembled in the reduced system matrices of the structure. Therefore, extra calculations are prevented for such components during the optimization process.

The reduced system matrices of the structure for each different design configuration are generated by 


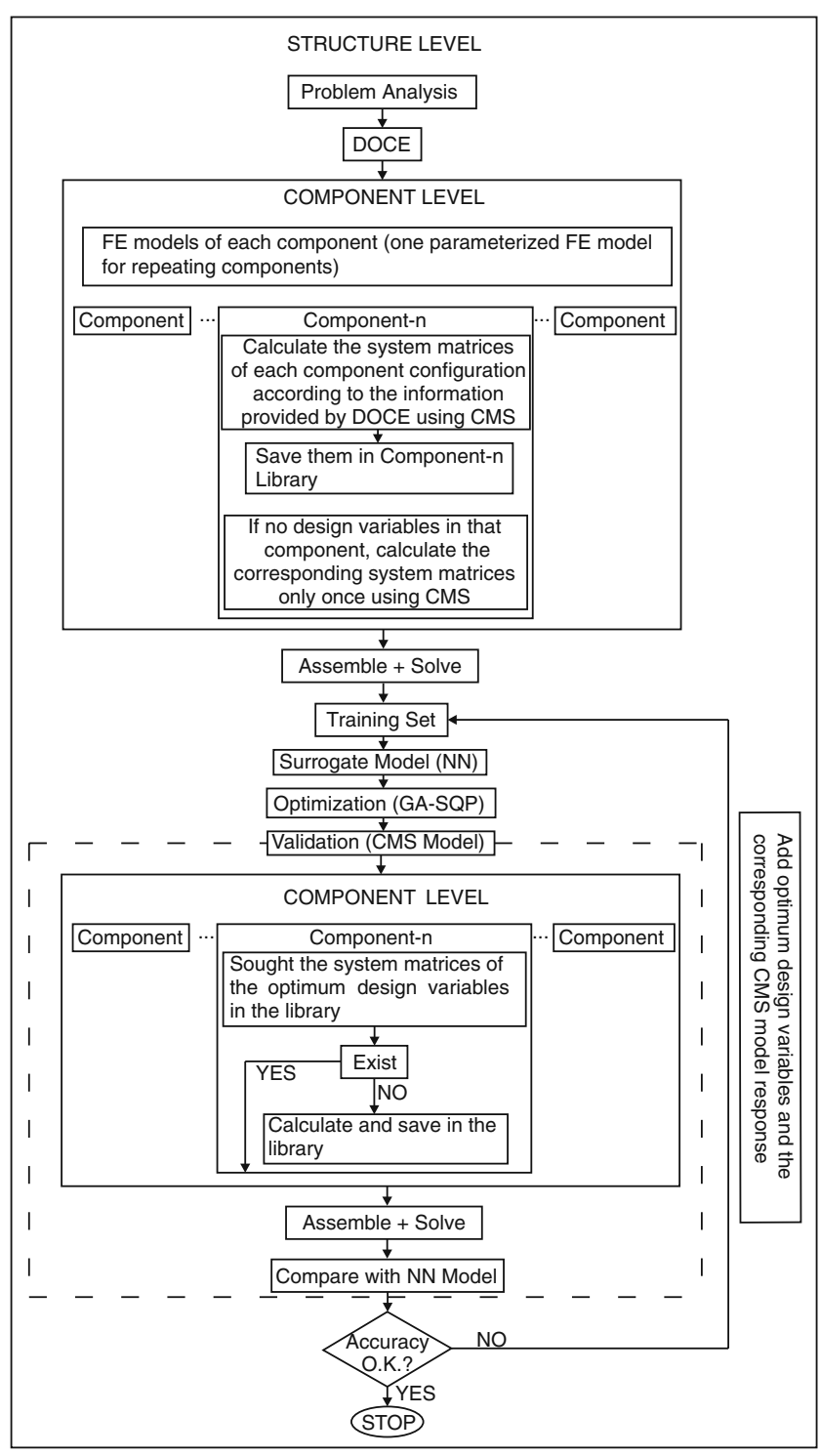

Fig. 1 The design optimization method

assembly and solved at the Structure Level. The required information is collected from the component libraries.

Using the generated training set, a surrogate model can be found by the BNNs which replaces the CMS based FE model in the optimization problem.

Next, the optimization is performed at the Structure Level using GA whose solution is provided as an initial point for SQP for finding an exact solution. Therefore, the chance of obtaining an exact global optimum solution is increased.

As mentioned earlier, only a limited number of sample points are selected at the beginning of the method. Since the obtained surrogate model is based on that limited amount of data, it may not represent the actual trend accurately. When the problem is optimized using that surrogate model, the attained results may not be reliable. Hence, it is very important to validate the response of the surrogate model with the response of the CMS based FE model. This is done at the end of the optimization step where the system matrices of the optimum design variables are sought in the corresponding component libraries at the Component Level. If not found, they are calculated and saved in the related libraries.

The assembly and the solution are performed at the Structure Level for calculating the response of the overall structure for calculated optimum design variables. If its response correlates with the response of the surrogate model, the scheme is stopped. Otherwise, it is an indication of a poor surrogate model. The optimum design variables and the corresponding structure response are added to the training set and the NNs are trained again to obtain a better surrogate model. The same procedure is followed until the relative error between the CMS based FE model result and the surrogate model result is small enough.

\section{Demonstration of the method}

For the demonstration of the proposed method, a structure resembling a fan inlet case was selected. The structure and its repeating component are illustrated in Fig. 2a, the physical parameters and the design variables of the component are shown in Fig. 2b. The thickness of the strut, $t_{\text {strut }}$, the inner ring, $t_{\text {inner }}$ and the outer ring, $t_{\text {outer }}$, of each component are selected as design variables and the components which have $n \frac{\pi}{2}, n=0, \ldots, 3$ rotational distance between each other are assumed to have the same design variables. In Fig. 2c, the identical colors represent the components that have the same design variables. Since there are 24 components on the structure and in every quarter the component is repeated, there exist 6 different components with 3 design variables where the components are illustrated via numbering in Fig. 2c. Thus, in total there are 18 design variables. The structure is a free-free structure (there are no defined boundary conditions on the structure).

With the same design variables, all the substructures are identical in the local coordinates. Thus, the CraigBampton transformation, stiffness and mass matrices of each substructure are all the same. Consequently, the reduced FE model of the entire structure can be obtained using the reduced FE model of one repeated component. 


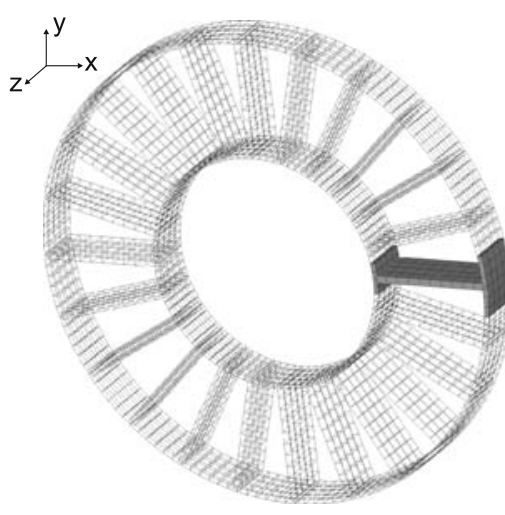

(a) The selected structure and its repeating component

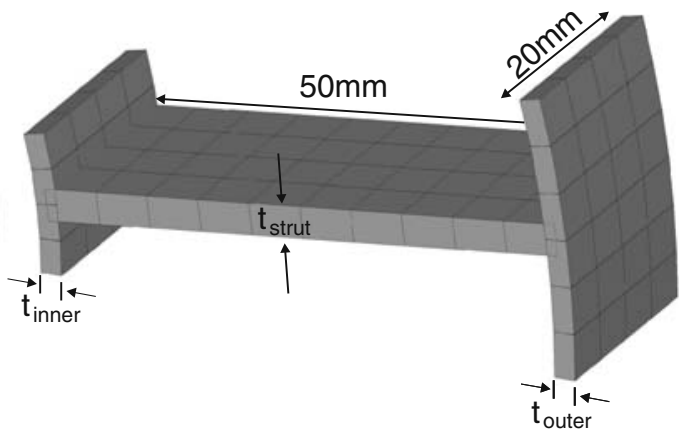

(b) The physical parameters and the design variables of one component.

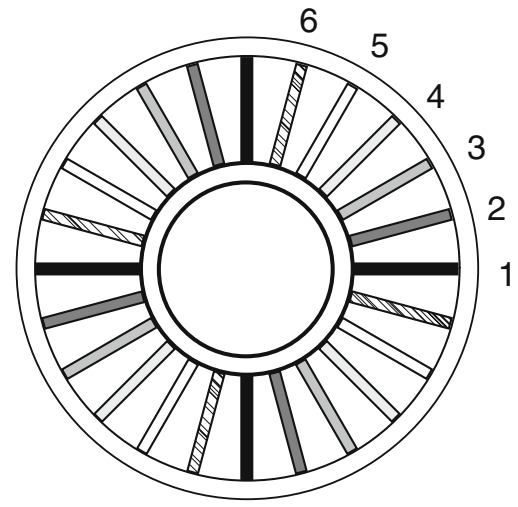

(c) Matching colors represent the components with the same design variables.

Fig. 2 The selected structure for the demonstration of the strategy (a-c)

In this study, the reduced system matrices of a selected component were generated using the CraigBampton method for different component configurations using the commercial FE software ANSYS. Assigning these matrices to the rest of the substructures by multiplying them with the corresponding rotation matrices, assembling the substructure system matrices for each design configuration and solving the eigenvalue problem was performed in MATLAB. In the FE model, Shell181 elements were used which are suitable for analyzing thin to moderately thick shell structures. Each element has 6 d.o.f at each node which are the translations and rotations on the $x^{-}, y_{-}, z$-axes. The in-plane vibrations of the ring structure are the only concern for this problem. Therefore, the rotations on $\mathrm{x}-, \mathrm{y}$-axes and the translations in the $\mathrm{z}$-direction are suppressed in the element. The reduced model of the structure has 1560 d.o.f where each component has 65 d.o.f. The full FE model of the structure has 6840 d.o.f. The selected material properties are as follows: Young's modulus $(E)$ is $116 \mathrm{GPa}$., Poisson's ratio $(v)$ is 0.3 and the density $(\rho)$ is $4500 \mathrm{~kg} / \mathrm{m}^{3}$.

In the initial design, the components have an inner ring thickness of $\left(t_{\text {inner }}^{i}, i=1,2, \ldots, 6\right) 2 \mathrm{~mm}$, a strut thickness of $\left(t_{\text {strut }}^{i}, i=1,2, \ldots, 6\right) 3 \mathrm{~mm}$, an outer ring thickness of $\left(t_{\text {outer }}^{i}, i=1,2, \ldots, 6\right) 2 \mathrm{~mm}$ where $i$ stands for the component number. The total mass of the initial model was $0.4936 \mathrm{~kg}$ and the $5^{\text {th }}$ natural frequency ( $2^{\text {nd }}$ bending frequency) was $702.23 \mathrm{~Hz}$ with a mode shape illustrated in Fig. 4a. Because this is a free-free structure, the first three modes are rigid body modes.

For the optimization problem the total mass of the entire structure is to be minimized by adjusting the defined thicknesses while increasing the $5^{\text {th }}$ natural frequency from $702.23 \mathrm{~Hz}$ to $750 \mathrm{~Hz}$ and preserving the $5^{\text {th }}$ mode shape of the initial design.

The optimization problem is formulated as follows:

$\min _{\mathbf{t}} \rho V(\mathbf{t})$

sbj. to $f_{5}=750$

$$
\begin{aligned}
& \text { MAC }_{5} \geq 0.9 \\
& 1 \leq t_{\text {inner }}^{i} \leq 5 \\
& 1 \leq t_{\text {strut }}^{i} \leq 5 \\
& 1 \leq t_{\text {outer }}^{i} \leq 5 \quad i=1, \ldots, 6 .
\end{aligned}
$$

In (11), $V$ represents the volume of the entire structure which is a function of the thicknesses $t$. In order to keep the mode shape of the initial design the same, the Modal Assurance Criterion (MAC) was used.

The MAC is a scalar value between 0 and 1, representing the correlation number between two mode shapes. A MAC value near 1 indicates a high degree of correlation between two mode shapes. If $\mathbf{u}$ and $\mathbf{v}$ are assumed to be two eigenvectors their MAC value is given by:

$\operatorname{MAC}(\mathbf{u}, \mathbf{v})=\frac{(\mathbf{u} \cdot \mathbf{v})^{2}}{\|\mathbf{u}\|^{2}\|\mathbf{v}\|^{2}}$

where ' ' represents the dot product. It is clear that MAC is nothing but the square of the cosine of the angle between two vectors.

Since the structure under consideration has a rotational symmetry in its geometry, there may exist multiple eigenvectors corresponding to the same eigenvalue. 
Table 1 Summary of the optimization problem

Initial design variables $t_{\text {inner }}$

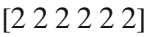

Initial design variables $t_{\text {strut }}$

$\left[\begin{array}{lllllll}3 & 3 & 3 & 3 & 3 & 3\end{array}\right]$

Initial design variables $t_{\text {outer }}$

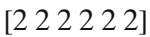

Optimum design variables $t_{\text {inner }}$

[ [ $\left.\begin{array}{lllllll}3.5 & 2.7 & 2.9 & 3 & 4 & 3.9\end{array}\right]$

Optimum design variables $t_{\text {strut }}$

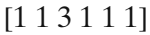

Optimum design variables $t_{\text {outer }}$

\# of designs in the library (initial)

[1 11111111$]$

\# of designs in the library (final)

180

Total \# of iterations

867

185

$0.49 \mathrm{~kg}$

$0.29 \mathrm{~kg}$

0.99

Optimum mass

$750 \mathrm{~Hz}$

Final $f_{5}(\mathrm{NN})$

$750.33 \mathrm{~Hz}$

These eigenvectors are linearly independent and can be combined in order to give an orthogonal basis. Any linear combination of the orthogonal basis vectors also have the same eigenvalue (Geradin and Rixen 1994).

Therefore in the solution strategy, instead of using the mode shape of the $5^{\text {th }}$ natural frequency directly in the MAC formulation (12), first, the uniqueness of the $5^{\text {th }}$ natural frequency is inspected. If it is unique, the corresponding eigenvector is used in (12). If there exist multiple eigenvectors that correspond to the $5^{\text {th }}$ natural frequency, then these are gathered and orthogonalized using QR decomposition in order to obtain an orthogonal basis. Afterwards, the most complying vector with the $5^{\text {th }}$ eigenvector of the initial design is sought in a subspace spanned by this orthogonal basis, and this latter vector is applied in (12). Details of the formulation are presented in Appendix.

Two NN surrogate models with 25 hidden layer neurons were employed in the optimization problem which represent $f_{5}$ and $\mathrm{MAC}_{5}$. For generating a training set for surrogate modeling only the model of one repeating component was used. Since each component has 3 design variables, first a $180 \times 3$ DOCE set, $\mathbf{D}_{1}$, was generated where each row stands for a new component design and each column of the set corresponds to the varying thicknesses of the inner ring, the strut and the outer ring, respectively. Then the Craig-Bampton stiffness and mass matrices were calculated for each component design and stored in a library for later use. The next step is to gather system matrices of the structure using this information. Since there are 6 different components in a structure with 3 varying design variables, the rows of $\mathbf{D}_{1}$ were permuted for each component and then gathered to generate a sample

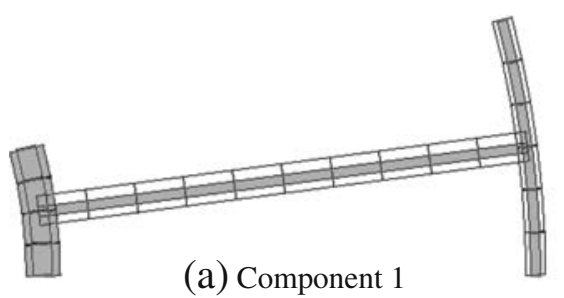

(a) Component 1

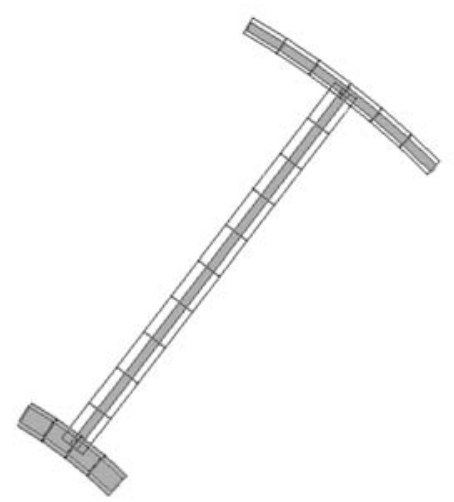

(d) Component 4

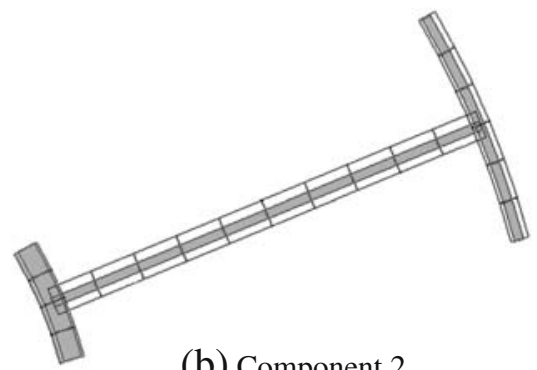

(b) Component 2

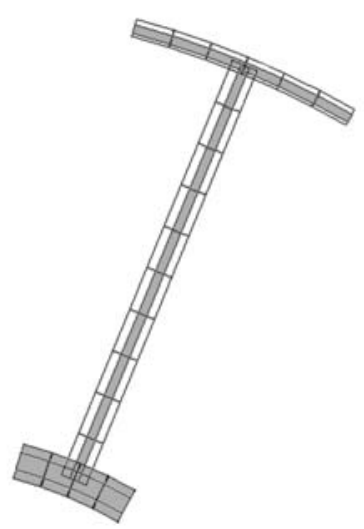

(e) Component 5

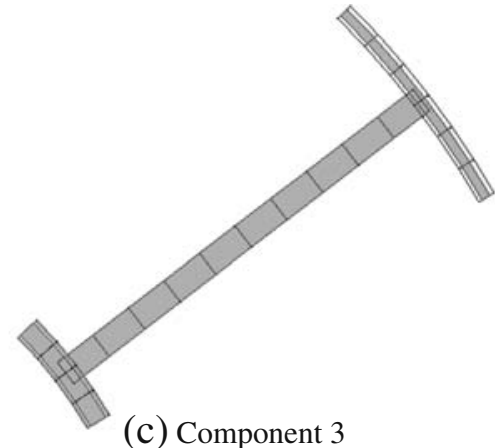

(c) Component 3

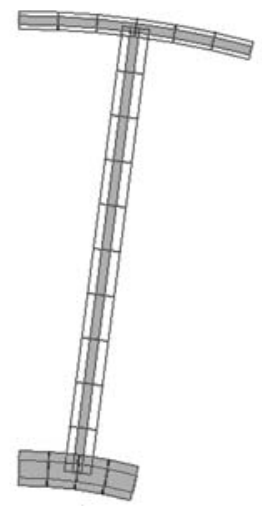

(f) Component 6

Fig. 3 The initial and the final design of the components. The grey color corresponds to the final design (a-f) 


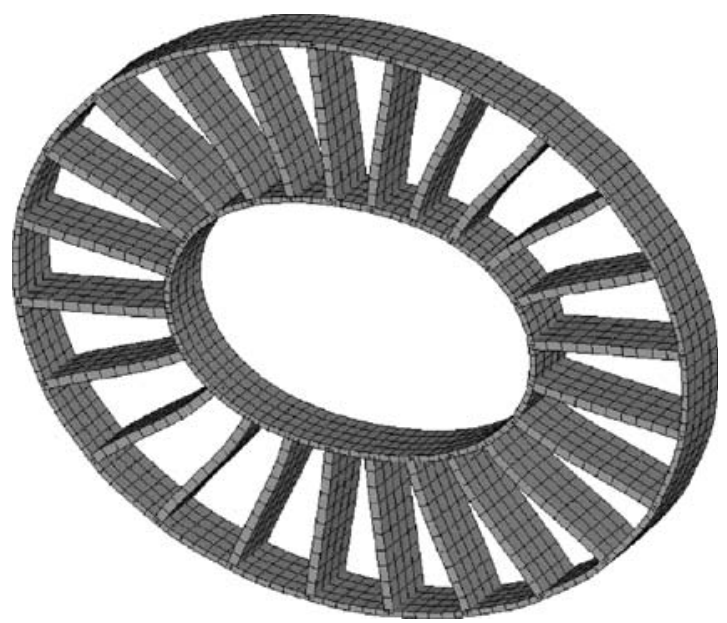

(a) Initial design

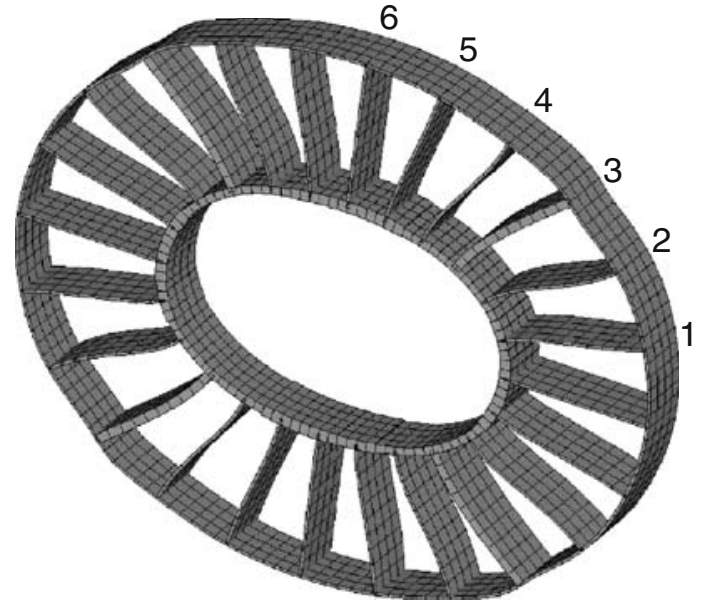

(b) Optimum design

Fig. 4 The initial and the optimum designs and the corresponding $5^{\text {th }}$ mode shapes $(\mathbf{a}, \mathbf{b})$

set, $\mathbf{D}_{\mathrm{T}}$, for the entire structure which has a size of $180 \times 18$. Therefore each row of $\mathbf{D}_{T}$ represents one possible design configuration of a structure. Due to the fact that the system matrices of each component configuration have already been calculated and stored in a library, we only need to call the system matrices from the library, multiply them by the corresponding rotation matrices for transforming them to their global coordinates, assemble them and solve an eigenvalue problem. At the end of the solution process a set of eigenvalues representing the $5^{\text {th }}$ natural frequency and the corresponding eigenvectors are obtained. $\mathbf{D}_{T}$ and the frequency set are used for training the NN which replaces $f_{5}$. The computed eigenvectors were used to calculate the MAC values. Afterwards, $\mathbf{D}_{T}$ and the MAC set were used for training the NN which represents $\mathrm{MAC}_{5}$.

In the validation step, when the relative error between the CMS based FE model and the surrogate models is smaller than 0.005 for each case, the procedure is stopped, otherwise it is continued until the relative error is smaller than the desired value.

The results of the optimization problem are summarized in Table 1. The initial and the final design of each component are illustrated in Fig. 3. The $5^{\text {th }}$ mode shapes for the initial and the optimum design are presented in Fig. 4. In the final design, a $40 \%$ reduction in total mass was achieved. Investigating closely it can be observed that the inner ring thickness of each component is greater than its initial value. This is because the inner ring thickness has a dominant effect on the stiffness whereas its contribution to the total mass of the structure is less compared to the other design variables. Thus, it guarantees the satisfaction of the constraint on the $5^{\text {th }}$ natural frequency. On the other hand, the strut and the outer ring thicknesses have a primary effect on the total mass. That is why in the final design the corresponding variables are at their minimum permissible values in order to minimize the total mass, accept the strut thickness of the $3^{\text {rd }}$ component. This remains the same and ensures the MAC constraint of the problem.

Since the chosen model is not sufficiently complex, the number of component and full system calculations are compared instead of computation times. The optimization process was concluded at the end of 185 iterations. The final component library involves 867 different component configurations where the component system matrices have the size of $65 \times 65$. If the optimization had been performed using the full model of the structure, the full FE model would have been called 365 times, where the system matrices have the size of $6840 \times 6840$.

The difference between the size of the models becomes more pronounced when complicated structure models come into play which may cause a drastic computational difference.

\section{Conclusions}

Using surrogate models instead of FE models becomes a common practice in most of the design optimization processes. On the other hand, FE models are still required to gather a training set for surrogate modeling. For certain applications using CMS in FE analysis may cause a lot of reduction in computation time. In this research, the benefits of CMS is utilized for the 
optimization of structures which have repeating patterns. In the proposed method, the analysis is decomposed and reduced using the Craig-Bampton method. Additionally, only one repeating pattern is modeled whose calculated system matrices are utilized for the rest of the repeating patterns. Therefore extra calculations for obtaining the system matrices of each repeating pattern is avoided which further decreases the computation time. Backpropagation NNs with Bayesian regularization are employed as the surrogate models. The strength of this method shows itself when there is no information about the nonlinearity of the input-target relationship and the training data involves numerical noise. Optimization is performed on the structure level without any decomposition. The two step optimization scheme increases the chance of finding an exact global optimum. The results indicate that the method is performing satisfactorily and very promising for real life applications.

It is important to point out that employing more effective strategies, such as the method of Rixen (Rixen 2004), for the assembly of the components may improve the applicability of the method. Another point for research might be the study of optimization problems involving large number of design parameters.

\section{Appendix}

Finding the most complying eigenvector with the initial designs eigenvector of interest and their MAC value:

Let us represent the $\mathrm{k}^{\text {th }}$ eigenvector of the initial design with $\mathbf{u}$. Assume that $\left\{\mathbf{v}_{1}, \mathbf{v}_{2}, \ldots, \mathbf{v}_{m}\right\}$ are the orthogonalized eigenvectors of the current design which correspond to the $\mathrm{k}^{\text {th }}$ eigenvalue $\omega_{k}$ and span the subspace $\mathbf{V}$.

The most complying eigenvector $\mathbf{y}_{c}$ in the subspace $\mathbf{V}$ with $\mathbf{u}$ and their MAC value are calculated using the formulas:

$\mathbf{y}_{c}=\frac{\mathbf{v}_{\mathbf{1}}^{\mathrm{T}} \mathbf{u}}{\left\|\mathbf{v}_{1}\right\|^{2}} \mathbf{v}_{1}+\ldots+\frac{\mathbf{v}_{\mathbf{m}}^{\mathrm{T}} \mathbf{u}}{\left\|\mathbf{v}_{m}\right\|^{2}} \mathbf{v}_{m}$

$\operatorname{MAC}\left(\mathbf{u}, \mathbf{y}_{c}\right)=\operatorname{MAC}\left(\mathbf{u}, \mathbf{v}_{1}\right)+\ldots+\operatorname{MAC}\left(\mathbf{u}, \mathbf{v}_{m}\right)$.

The derivation of the formulas are as follows:

The Degeneracy Theorem (Geradin and Rixen 1994) states that any vector contained in the subspace $\mathbf{V}$ also belongs to the same eigenvector $\boldsymbol{\omega}_{k}$.

Finding the vector in $\mathbf{V}$ which complies with $\mathbf{u}$ the most:

Let $\mathbf{y}=c_{1} \mathbf{v}_{1}+c_{2} \mathbf{v}_{2}+\ldots+c_{m} \mathbf{v}_{m}$ be a vector that lies in $\mathbf{V}$ where $c_{i}, i=1,2, \ldots, m$ are scalars. The distance between $\mathbf{y}$ and $\mathbf{u}$ can be defined using Euclidean distance as:

$$
\begin{aligned}
\|\mathbf{y}-\mathbf{u}\|^{2}= & \left\|c_{1} \mathbf{v}_{1}+c_{2} \mathbf{v}_{2}+\ldots+c_{m} \mathbf{v}_{m}-u\right\|^{2} \\
= & c_{1}^{2}\left\|\mathbf{v}_{1}\right\|^{2}+c_{2}^{2}\left\|\mathbf{v}_{2}\right\|^{2}+\ldots+c_{m}^{2}\left\|\mathbf{v}_{m}\right\|^{2} \\
& -2 c_{1} \mathbf{v}_{1}^{\mathrm{T}} \mathbf{u}-\ldots-2 c_{m} \mathbf{v}_{m}^{\mathrm{T}} \mathbf{u}+\mathbf{u}^{\mathrm{T}} \mathbf{u}
\end{aligned}
$$

If the distance between $\mathbf{y}$ and $\mathbf{u}$ is minimized with respect to the coefficients $c_{i}, i=1,2, \ldots, m$, it is possible to obtain the vector which has a high correlation with u. In order to accomplish this:

$$
\begin{array}{cc}
\frac{\partial\|\mathbf{y}-\mathbf{u}\|^{2}}{\partial c_{1}}=2 c_{1}\left\|\mathbf{v}_{1}\right\|^{2}-2 \mathbf{v}_{1}^{\mathrm{T}} \mathbf{u}= & 0 \\
\vdots & \vdots \\
\frac{\partial\|\mathbf{y}-\mathbf{u}\|^{2}}{\partial c_{m}}=2 c_{m}\left\|\mathbf{v}_{m}\right\|^{2}-2 \mathbf{v}_{m}^{\mathrm{T}} \mathbf{u}=0
\end{array}
$$

Using (14); $c_{1}, c_{2}, \ldots, c_{m}$ are calculated as follows:

$c_{1}=\frac{\mathbf{v}^{\mathrm{T}} \mathbf{u}}{\left\|\mathbf{v}_{1}\right\|^{2}}, \ldots, c_{m}=\frac{\mathbf{v}_{\mathbf{m}}^{\mathrm{T}} \mathbf{u}}{\left\|\mathbf{v}_{m}\right\|^{2}}$.

Hence, in the subspace $\mathbf{V}$ the vector $\mathbf{y}_{c}$;

$\mathbf{y}_{c}=\frac{\mathbf{v}_{\mathbf{1}}^{\mathrm{T}} \mathbf{u}}{\left\|\mathbf{v}_{1}\right\|^{2}} \mathbf{v}_{1}+\ldots+\frac{\mathbf{v}_{\mathbf{m}}^{\mathrm{T}} \mathbf{u}}{\left\|\mathbf{v}_{m}\right\|^{2}} \mathbf{v}_{m}$

has a high correlation with the vector $\mathbf{u}$ where the correlation is calculated using (12) as:

$\operatorname{MAC}\left(\mathbf{u}, \mathbf{y}_{c}\right)$

$$
\begin{aligned}
& =\frac{\left(\mathbf{u}^{\mathrm{T}} \mathbf{y}_{c}\right)^{2}}{\|\mathbf{u}\|^{2}\left\|\mathbf{y}_{c}\right\|^{2}}=\frac{\left(\mathbf{u}^{\mathrm{T}}\left(\frac{\mathbf{v}_{1}^{\mathrm{T}} \mathbf{u}}{\left\|\mathbf{v}_{1}\right\|^{2}} \mathbf{v}_{1}+\ldots+\frac{\mathbf{v}_{\mathbf{m}}^{\mathrm{T}} \mathbf{u}}{\left\|\mathbf{v}_{m}\right\|^{2}} \mathbf{v}_{m}\right)\right)^{2}}{\|\mathbf{u}\|^{2}\left\|\frac{\mathbf{v}_{1} \mathbf{T}^{\mathrm{T}}}{\left\|\mathbf{v}_{1}\right\|^{2}} \mathbf{v}_{1}+\ldots+\frac{\mathbf{v}_{\mathbf{m}} \mathrm{T}}{\left\|\mathbf{v}_{m}\right\|^{\mathrm{T}}} \mathbf{v}_{m}\right\|^{2}} \\
& =\ldots=\frac{\left(\mathbf{u}^{\mathrm{T}} \mathbf{v}_{1}\right)^{2}}{\|\mathbf{u}\|^{2}\left\|\mathbf{v}_{1}\right\|^{2}}+\ldots+\frac{\left(\mathbf{u}^{\mathrm{T}} \mathbf{v}_{m}\right)^{2}}{\|\mathbf{u}\|^{2}\left\|\mathbf{v}_{m}\right\|^{2}} \\
& =\operatorname{MAC}\left(\mathbf{u}, \mathbf{v}_{1}\right)+\ldots+\operatorname{MAC}\left(\mathbf{u}, \mathbf{v}_{m}\right) .
\end{aligned}
$$

Open Access This article is distributed under the terms of the Creative Commons Attribution Noncommercial License which permits any noncommercial use, distribution, and reproduction in any medium, provided the original author(s) and source are credited.

\section{References}

Akçay Perdahcioğlu D, van der Hoogt P, de Boer A (2007) Design optimization applied in structural dynamics. In: Proceedings of 1st international conference on artificial intelligence for industrial applications, pp 45-50

Conn A, Gould N, Toint P (1991) A globally convergent augmented lagrangian algorithm for optimization with general constraints and simple bounds. SIAM J Numer Anal 28(2):545-572

Craig R, Bampton M (1968) Coupling of substructures for dynamic analysis. AIAA 6(7):1313-1319 
Foresee F, Hagan M (1997) Gauss newton approximation to bayesian learning. In: IEEE trans. on neural networksproceedings of ICNN 97, pp 1930-1935

Geradin M, Rixen D (1994) Mechanical vibrations-theory and applications to structural dynamics. Wiley, New York

Gill P, Murray W, Saunders M, Wright M (1984) Procedures for optimization problems with a mixture of bounds and general constraints. ACM Trans Math Softw 10(3):282-298

Giunta A, Wojtkiewicz S Jr, Eldred M (2003) Overview of modern design of experiments methods for computational simulations. In: AIAA-2003-649, 41st aerospace sciences meeting and exhibit, Reno

Gould N, Conn A, Toint P (1997) A globally convergent lagrangian barrier algorithm for optimization with general inequality constraints and simple bounds. Math Comput 66(217):261-288

Hagan M, Demuth H, Beale M (1996) Neural network design, chapter 11. PWS, Boston

Han S (1977) A globally convergent method for nonlinear programming. J Optim Theory Appl 22(3):297-309

Hornik K (1989) Multilayer feedforward networks are universal approximators. Neural Netw 2:359-366
Hou G, Maroju V, Yang R (1995) Component mode synthesis based design optimization method for local structural modification. Struct Optim 10:128-136

Kumar R (2007) Genetic algorithm and direct search toolbox, version 2.1. MATLAB R2007a

Mackay D (1992) Bayesian interpolation. Neural Comput 4: 415-447

McKay M, Beckman R, Conover W (1979) A comparison of three methods for selecting values of input variables in the analysis of output from a computer code. Technometrics 21(2):239-245

Rixen D (2004) A dual craig-bampton method for dynamic substructuring. J Comput Appl Math 168(1-2):383-391

Sobieszczanski-Sobieski J (1989) Multidisciplinary optimization for engineering systems: achievements and potential. Technical memorandum N89-24508, NASA

Wind J (2005) Silent components fast, a global local optimization method for dynamic problems. Master's thesis, University of Twente

Wind J, Akçay Perdahcıoğlu D, de Boer A (2008) Distributed multilevel optimization for complex structures. Struct Multidisc Optim 36(1):71-81 